\title{
La formación de nuevos investigadores y el manejo de las alfabetizaciones informacional, cientíica y académica
}

\author{
Training new researchers and managing information, scientific and academic literacies
}

\author{
Tania G. Ortiz-Santana - Juan D. Machin-Mastromatteo - Javier Tarango
}

Tania G. Ortiz-Santana. Universidad Autónoma de Chihuahua, México. Es licenciada en Motricidad Humana por la Facultad de Ciencias de la Cultura Física y maestra en Innovación Educativa por la Facultad de Filosofía y Letras de UACH. Cursa actualmente el segundo semestre del doctorado en Educación, Artes y Humanidades en la misma universidad. Correo electrónico: taniag.ortiz.santana@gmail.com ORCID: https://orcid.org/0000-0003-2541-7403.

Juan D. Machin-Mastromatteo. Universidad Autónoma de Chihuahua, México. Es profesor investigador, miembro del Sistema Nacional de Investigadores, Nivel I, e integrante del Cuerpo Académico Consolidado UACH088 Estudios de la Información. Doctor en Ciencias de la Información y Comunicación. Ha publicado más de 50 artículos científicos, cinco libros y 15 capítulos de libro. Editor asociado de Digital Library Perspectives y miembro de los comités de IE Revista de Investigación Educativa de la REDIECH, The Journal of Academic Librarianship e Information Development. Correo electrónico: jmachin@uach.mx ORCID: https://orcid. org/0000-0003-4884-0474.

Javier Tarango. Universidad Autónoma de Chihuahua, México. Es profesor investigador y miembro del Sistema Nacional de Investigadores, Nivel II. Doctor en Educación y miembro del Instituto Agustín Millares Carlo de Documentación y Gestión de Informa-
Resumen

Este artículo relata el inicio de una investigación que se planteó con el fin de brindar apoyo a las instituciones de educación superior para incrementar sus niveles de producción científica, mediante la formación de nuevos investigadores. Busca identificar los entrecruces entre las subjetividades y la praxis de la investigación, a través de un estudio cualitativo con enfoque fenomenológico, que tiene el objetivo de conocer la experiencia de ser un investigador, encontrar cuáles son las características, aspectos de la personalidad, prácticas, habilidades, etapas de evolución y procesos de formación que tipifican a un individuo como investigador, además de llegar a definir qué significa ser un investigador, qué es investigar y qué praxis produce, cuál es el rol de las experiencias de los investigadores consolidados en la formación de nuevos investigadores y de qué manera los investigadores fungen como mentores y capacitadores. Los resultados que aquí se presentan corresponden a la primera fase de la investigación, en la que encontramos algunas de las habilidades y capacidades necesarias para que un investigador novel pueda trascender en el mundo de la investigación y publicación científica y académica.

Palabras clave: Educación para la investigación, formación de profesores, formación de investigadores, habilidades investigativas, educación superior.

Abstract
This article summarizes the beginning of a research that was started to support
higher education institutions in increasing their scientific production levels
through the training of new researchers. It seeks to identify the crossroads
among the research subjectivities and praxis through a qualitative study with a
phenomenological focus, with the objective of understanding the experience
of being a researcher, describing the characteristics, personality traits, practices,
skills, evolution stages and training processes that characterize an individual
as a researcher, as well as defining what it means to be a researcher, what is
conducting research and what kind of praxis it produces, what are the roles of


ción de la Universidad Carlos III de Madrid. Líder del Cuerpo Académico Consolidado UACH-088 Estudios de la Información. Sus líneas de investigación incluyen la evaluación de la producción y comunicación científica; gestión del conocimiento y la innovación, e identificación y desarrollo de la cultura informacional. Es árbitro de más de 30 revistas científicas internacionales. Correo electrónico: jtarango@uach.mx ORCID: https://orcid.org/00000002-0416-3400. consolidated researchers' experiences in the training of new researchers, and in what ways these researchers serve as mentors and trainers. The results presented here correspond to the first phase of the research, in which we found some of the skills and abilities that a novice researcher requires to transcend in the world of scientific and academic research and publication.

Keywords: Education for research, training of professors, training of researchers, research skills, higher education.

\section{INTRODUCCIÓN}

La presente investigación se planteó con el fin de brindar apoyo a las instituciones de educación superior (IES) para incrementar sus niveles de producción científica mediante la formación de nuevos investigadores. Además busca un cambio en los programas de formación de nuevos investigadores, mediante la implementación de nuevas metodologías que brinden oportunidades de formar investigadores de calidad, capacitados con competencias que los posicionen como productores de material científico de impacto.

Para las IES la formación de nuevos investigadores es una tarea de importante. La presente investigación, de la cual este artículo reporta resultados parciales, busca identificar los entrecruces entre las subjetividades y la praxis de la investigación. Se comprende la subjetividad como las habilidades, capacidades y características ideales de un investigador (González, 2019), y la praxis es la realidad a la que se enfrenta día a día para cumplir con los retos y roles de su profesión.

En relación con los ideales de la investigación, el Consejo Nacional de Ciencia y Tecnología (СОNACут) es el organismo que promueve la innovación y desarrollo tecnológico del país, a través de programas como el Sistema Nacional de Investigadores (SNI), que integra a los investigadores más productivos y busca fortalecer la investigación mediante la formación y consolidación de investigadores. Para los científicos, pertenecer al SNI representa ser parte de un círculo selecto de investigadores, que distingue a quienes realizan con mayor eficiencia su trabajo y a su vez realizan contribuciones importantes al conocimiento (Rodríguez, González y Maqueda, 2017). Por esta razón, en lo que respecta a México, el SNI marca las pautas a seguir por los investigadores a través de sus sistemas de evaluación para ingreso y permanencia, que incluyen requerimientos de publicación, docencia y gestión (Olivas y Musi, 2019). De acuerdo con Rodríguez, González y Maqueda (2017), “existen 177 universidades públicas, 249 institutos tecnológicos y 1835 instituciones de educación privada en México (...) durante dos décadas de estudio, únicamente 156 IES contribuyeron al conocimiento con al menos un artículo publicado en una revista ISI [sic]” (p. 216). Bajo el término ISI los autores citados se refieren a las revistas indizadas en el Science 
Citation Index Expanded o en el Social Science Citation Index. Ambos índices se actualizan anualmente en los Journal Citation Reports (JCR).

Para cumplir los requerimientos del SNI el investigador debe contar por lo menos con cinco artículos publicados en los últimos tres años (Hernández, 2019). Para cumplir con dichos requisitos e identificarse como investigador consolidado consideramos que el individuo deberá contar con habilidades de alfabetización informacional, científica y académica (AICA). Las AICA comprenden habilidades de escritura científica, metodológicas, tecnológicas, éticas y de gestión de la información, las cuales pueden variar dependiendo del área del conocimiento; por lo tanto, es necesario encontrar las habilidades comunes requeridas por los investigadores para desenvolverse exitosamente, independientemente de su área.

La investigación resulta de procesos de práctica y formación constantes, apoyados por las relaciones entre investigadores consolidados y nóveles. Tales interacciones buscan la adquisición del habitus científico, y en México una de las formas en que dichas interacciones se desarrollan es en las IES, a través de los programas de posgrado, mediante la conceptualización de la investigación como forma de comprender la vida, la cultura y el mundo (Mercado, Cernas y Nava, 2015).

El tutor es pieza clave para la formación de nuevos investigadores, y mediante un proceso de mimetización el investigador novel va adquiriendo las prácticas y hábitos que van creando un perfil de investigador. Las funciones del tutor incluyen a) acompañarlo durante el proceso de aprendizaje y crecimiento como investigador; b) conocer, favorecer y evaluar las etapas y avances de la investigación mediante seminarios, talleres, congresos, diálogo, resolución de dudas y toma de decisiones; c) valorar el cumplimiento del rigor científico y metodológico en la investigación (Torres-Frías, Moreno-Bayardo y Jiménez-Mora, 2018); e) potenciar e inculcar aspectos relacionados con la metodología, escritura científica y tradiciones de la disciplina. La elaboración de proyectos de tesis permitirá que todas las funciones mencionadas ocurran y además habilitará al investigador novel para que adquiera el discurso disciplinario, la capacidad de argumentación y el dominio de las estructuras retóricas necesarias, así como la genealogía de su campo temático. He aquí la necesidad de contar con investigadores con suficiencia para ser formadores de nuevos investigadores, la cual pueden demostrar mediante la publicación de trabajos en revistas científicas de alto nivel, que representan los cánones del conocimiento actual (Vessuri, 2007).

En la presente investigación, las AICA son las habilidades indispensables requeridas por un investigador y tienen sus bases en la alfabetización informacional (ALFIN), entendida como la capacidad de pensar críticamente, además de conformar un conjunto de habilidades necesarias para realizar tareas relacionadas con la información, como: encontrarla, interpretarla, analizarla, gestionarla, comunicarla, almacenarla y compartirla (Cooan, Geekie, Goldstein, Jeskins, Jones, Macrae-Gibson, Secker y Walton, 2020). 
En la presente investigación unificamos diferentes alfabetizaciones bajo el término de AICA, a fin de englobar las necesidades y procesos informacionales, además de los componentes científicos y académicos necesarios para desarrollar una investigación y elaborar publicaciones científicas, lo cual también implica la suficiencia para manejar el discurso disciplinar y realizar las tareas académicas de formación de recursos humanos. El investigador que haya apropiado las AICA podrá dinamizar sus paradigmas epistémicos, lo cual implica cambios en su forma de pensar y actuar, por lo cual es necesario comprender la importancia de la interdisciplinariedad en la investigación (Pinto y Uribe-Tirado, 2016).

Para convertirse en investigador consagrado, el sujeto requiere pasar por un arduo proceso educativo de formación, dentro del cual existe un entrecruce entre las subjetividades de la investigación y la praxis. Esto implica la presencia de sistemas de evaluación, reglamentos y tiempos para la publicación de resultados de investigación, actividades de docencia, formación de recursos humanos y labores administrativas, para lo cual los investigadores emplean su praxis, apoyada por las AICA. Por lo tanto, es importante que las IES mexicanas cuenten con docentes capacitados en las AICA, que puedan desempeñarse como investigadores de calidad, idealmente pertenecientes al SNI, para incrementar la calidad y cantidad de la producción científica de las instituciones. Dicha mejoría se reflejaría directamente en el posicionamiento institucional y nacional dentro de los rankings universitarios. A nivel nacional es importante la producción de capital intelectual para el crecimiento y el desarrollo, pero tratándose de un bien tan intangible como el conocimiento, la formación de investigadores es primordial, ya que una fortaleza para el país es su capacidad de generar conocimiento, para obtener una emancipación y crecimiento intelectual nacional, ya que en el estudio de Rodríguez, González y Maqueda (2017) se muestra que en lo que respecta a México existen pocas universidades orientadas a la investigación y los mejores índices de producción se encuentran ubicados dentro de diez instituciones educativas, como es el caso de la UNAM, Cinvestav, IPN, ya que dentro de estas existe un entorno competitivo que busca mantener y mejorar la calidad de la investigación.

\section{Metodología}

La presente investigación se está realizando actualmente mediante una metodología cualitativa, a través de un enfoque fenomenológico. Tal tipo de estudio busca conocer la estructura de una experiencia y su finalidad es comprender un fenómeno desde la perspectiva personal de cada sujeto, a través de un análisis profundo y detallado de los participantes (Pickard, 2013). La elección del enfoque fenomenológico fue motivada al observar que el empleo de otras metodologías en investigaciones relacionadas con la enseñanza de AICA no resultan necesariamente en estudios a nivel del individuo, ya que cada investigador podría tener una visión única de su profesión y desarrollo como investigador. 
Consideramos importante indagar sobre el objeto de estudio empleando la fenomenología, ya que nos podría permitir recabar datos más profundos sobre el fenómeno de la enseñanza de AICA y el desarrollo de los investigadores, para así desarrollar métodos alternativos para la formación de investigadores. El análisis fenomenológico se presenta por medio de una narrativa descriptiva de datos detallados, debido a que cobra preponderancia entender a profundidad las subjetividades de los investigadores con relación a la praxis de hacer investigación.

En esta investigación buscamos encontrar cuáles son las características, aspectos de la personalidad, prácticas, habilidades, etapas de evolución y formación que tipifican a un individuo como investigador, además, qué significa ser un investigador, qué es investigar y qué praxis produce, cuál es el rol de las experiencias de los investigadores consolidados en la formación de nuevos investigadores y de qué manera los investigadores fungen como mentores y capacitadores. Adicionalmente, esta investigación indagará sobre las innovaciones metodológicas requeridas para fortalecer la formación y desarrollo de nuevos investigadores, dando preponderancia a sus AICA. Para responder a estos planteamientos, esta investigación se desarrollará a través de cuatro fases.

- Fase I. Entrevistas preliminares: creación de entrevista semiestructurada, de 26 preguntas, aplicada a docentes investigadores consolidados de la Facultad de Filosofía y Letras de la Universidad Autónoma de Chihuahua, miembros del Nivel I y II del SNI, experimentados en el proceso de formación de nuevos investigadores, pertenecientes a diferentes áreas disciplinares.

- Fase II. Entrevistas: se ampliará a docentes pertenecientes a todas las Facultades, se tomará en cuenta a por lo menos dos miembros de cada una de las siete áreas del SNI.

- Fase III. Intervención: conformada por dos etapas, cada una tendrá la duración de un semestre escolar (agosto-diciembre y enero-junio). Se llevará a cabo mediante un curso de AICA, aplicado a docentes de la Universidad Autónoma de Chihuahua (UACH) que quieran capacitarse para ser investigadores, mediante actividades para el manejo de escritura o redacción científica, manejo de manuales de estilo, arquitectura del documento, estado del arte y metodología.

- Fase IV. Monitoreo: constará de un monitoreo constante de los sujetos que recibirán el curso, mediante un seguimiento de su productividad científica a lo largo de un año, para observar si hubo algún avance en sus carreras como investigadores.

El resto del presente artículo se centra en dar a conocer los resultados de la primera fase de la investigación, basada en entrevistas preliminares. Para la aplicación de las entrevistas preliminares se seleccionaron dos investigadores pertenecientes a la UACH, miembros del SNI (niveles I y II), quienes cuentan con múltiples publica- 
ciones en revistas científicas de corriente principal y participan constantemente en procesos de formación de nuevos investigadores.

La entrevista aplicada fue semiestructurada, originalmente constaba de 26 preguntas base, como por ejemplo: “a) ¿Como se involucró en la investigación?”, “b) ¿Qué significa ser investigador?” y “c) ¿Cómo fue su experiencia de formarse como investigador?", las cuales aumentaron al momento de la aplicación, debido a la necesidad de puntualizar algunos detalles y aspectos de la formación de nuevos investigadores, como sería el caso de pedir a los entrevistados que relataran sus experiencias en la formación como investigadores, con preguntas como: "a) ¿Quién fue su director de tesis?”, “b) ¿Dónde realzó sus estudios de posgrado?”, “c) ¿Dónde labora actualmente?” y “d) ¿Qué es lo que le gusta más de su trabajo?”. Cada entrevista tuvo una duración de una hora y treinta minutos, durante los cuales se discutieron aspectos relacionados con la evolución de los entrevistados como investigadores, la influencia de sus tutores, cuerpos académicos y formas de pensar de cada uno.

Para desarrollar el estudio y por la necesidad de tener un análisis profundo y minucioso de la información es relevante mencionar que los estudios cualitativos de un solo sujeto tienen validez cuando es necesario realizar un análisis a profundidad de la información en áreas poco exploradas, en estos casos la opinión de una sola persona puede ser de mucha utilidad para comprender el contexto a explorar (Boddy, 2016). En este caso, al tratarse de un estudio preliminar de recolección de datos de los investigadores más productivos de la universidad, se buscó la aplicación de una cantidad pequeña de entrevistas bajo el razonamiento anterior y con el fin de obtener el grado de detalle necesario para la creación del instrumento final a partir de las preguntas realizadas en la entrevista preliminar y para realizar un análisis lo suficientemente profundo de las respuestas obtenidas. Esto se realiza para agotar la información de las fuentes, la cual es la finalidad principal de la investigación cualitativa.

Para el análisis de las entrevistas se procedió con la transcripción de sus grabaciones en audio, luego se realizó un proceso de codificación abierta y se crearon categorías según fueran emergiendo los temas principales durante las entrevistas. De esta manera se crearon cinco categorías principales: a) características del investigador, b) influencia, c) habilidades, d) formación de nuevos investigadores y e) evolución como investigador.

\section{Resultados}

A continuación se resumen las opiniones de los sujetos entrevistados con relación a la formación de nuevos investigadores, utilizando los códigos D1 y D2 para identificar y diferenciar las afirmaciones de cada entrevistado.

Es necesario formar nuevos investigadores para incidir en cambios, generar reflexiones, aportar algo para solucionar alguna problemática, para incidir en la formación de otras personas, mediante la formación de canales que vinculen a la educación con las necesidades del sector educativo. 
Aportar reflexiones para la mejora es una oportunidad de aportar algo para solucionar alguna problemática, para generar opinión, para incidir en la formación de otras personas [D1].

Para el crecimiento del país, mediante la generación de bienes de conocimiento, los países en desarrollo son dependientes de los países ricos en cuestiones de conocimiento, usamos el APA, que es americano, publicamos en revistas en inglés de cualquier país más rico que nosotros, porque no somos capaces de generar bienes y servicios de información y de conocimiento. El punto central es generar conocimiento, entonces la investigación y la formación de investigadores tendría que ser una contribución macrosocial, la emancipación de los pueblos en la dependencia del conocimiento de otros [D2].

Las características y aspectos de la personalidad son las que tienen que ver con redacción, manejo de las metodologías del campo, ya que cada área temática se diferencia por una manera específica de construir el conocimiento, poseer ciertas habilidades sociales para auto publicitarse [D1].

Es necesario tener gusto y disposición, posibilidades de llegar, acatar órdenes, capacidad de trabajo en pares y equipos. Desprendimiento, manejo de buenas fuentes de consulta, competencias lingüísticas, de escritura, capacidad de plasmar ideas en su idioma y en otro. Competencias tecnológicas, creatividad, innovación y transdisciplinariedad. Ser investigador no es una pose, es un conjunto de acciones sistemáticas cuyo reflejo son las publicaciones científicas, es una persona que goza de conocimientos metodológicos para afrontar, estudiar y profundizar una temática y encontrar una solución viable, teórica o práctica [D2].

Como síntesis de los temas conversados con los entrevistados, podemos afirmar que los investigadores se forman mediante la práctica. Los programas educativos sientan las bases, les piden que publiquen, hacen que los estudiantes tomen en serio los procesos de investigación, los cuales inician con retos como la consolidación de un planteamiento de un problema de investigación, luego se introducen poco a poco en el campo de investigación, hasta llegar a un punto en el que pueden publicar solos, sin depender del tutor.

Con relación a la praxis de la investigación, los docentes se vuelven investigadores a causa de las exigencias institucionales, para mantenerse en la vida académica. Ser docente investigador consta de actividades que giran en torno a los estudiantes, como la tutoría de tesis y la participación en proyectos de investigación. Se hace investigación para cumplir, para tener puntos para CONACут y otras evaluaciones como la "beca al desempeño", típica de las universidades públicas. La investigación se vuelve crediticia, además, cuando la persona ya es miembro del SNI y recibe una compensación económica, se vuelve una necesidad mantenerse en esto.

El tutor influye en la formación de nuevos investigadores mediante su participación como director de tesis y catedrático en los seminarios de maestría o doctorado. Este asesor marca las pautas, diciendo qué pasos seguir, pero cuando se han establecido los criterios generales, va dejando que cada quien busque su rumbo. Un investigador se considera como consolidado cuando participa en actividades de formación de recursos humanos, principalmente en posgrado, además publica los resultados de sus proyectos de investigación ya sea en libros, ponencias y artículos, además de la 
participación en congresos, dictaminación de artículos o capítulos de libros, mediante su interacción y trabajo en redes.

\section{DISCUSIÓN}

Dentro del proceso para la formación de nuevos investigadores los aspectos de la personalidad son sumamente importantes, ya que para que un sujeto logre trascender en el mundo de la investigación es necesario que posea características como: ambición, compromiso, disposición, capacidad de sobreponerse a sus prejuicios y potencial. Además, para el tránsito por este arduo proceso es necesario que el investigador novel cuente con alguna motivación o interés, y dicho interés puede motivarse por la obtención de un mejor puesto laboral o a la espera de la obtención de alguna remuneración económica (Olivas y Musi, 2019).

Más allá de que la persona requiera contar con determinadas características, también necesita contar ciertas habilidades. El candidato deberá contar con una gama de experiencias previas, que no necesariamente se asocian con la investigación pero que le serán de ayuda en su proceso de adquisición de habilidades para la investigación, tales como la autogestión y un desarrollo adecuado de AICA, necesarias para alcanzar un adecuado manejo temático de su área, así como las competencias necesarias para la búsqueda y categorización de la información, todas ellas necesarias en la labor de la investigación.

Hemos mencionado con anterioridad que el tutor es una pieza clave para el desarrollo de un investigador, ya que es quien marca las pautas a seguir por el investigador novel e influencia su manera de pensar y actuar (Mercado, Cernas y Nava, 2015). Todo el proceso de formación como investigador pasa por un espectro de fases, ordenadas de la primera a la última, de la siguiente manera: a) desconocimiento; b) dependencia, ciertamente necesaria del tutor; c) involucramiento, y d) emancipación. Es necesario poner atención de cerca al proceso de formación y a los aspectos por los cuales se ve influenciado (Vessuri, 2007).

El proceso de formación de un nuevo investigador tiene sus bases en la influencia, principalmente de su tutor, para la adquisición del habitus científico. Pero también es necesario tomar en cuenta la influencia del entorno del investigador novel, las presiones impuestas por la institución a la que pertenece, la convivencia constante con otros investigadores y, lo más importante, la influencia de sus estudiantes, ya que, como se ha mencionado, en nuestro país las labores de los docentes de las IES parten en sus ejes fundamentales de acción (docencia, investigación y extensión), en los que las direcciones de tesis cumplen un papel fundamental. 


\section{CONCLUSIÓN}

En lo que respecta a la formación de nuevos investigadores, es innegable la influencia de los sistemas de evaluación como el SNI, que marcan las pautas del desarrollo de la subjetividad del investigador, las condiciones hegemónicas, impactando incluso el desarrollo de programas educativos, en formas generalmente positivas. Es necesario mejorar los procesos de desarrollo de capital intelectual para fortalecer los indicadores de producción científica, investigación e innovación del país, a través de la generación de bienes y productos de conocimiento propios, para poder generar una emancipación cultural. La formación de nuevos investigadores es de suma importancia para el desarrollo de ciudadanos más conscientes y críticos, quienes a través de una adecuada formación en las AICA podrán dar solución a las problemáticas de su entorno. En la primera fase de la investigación reportada en este artículo se realizó un primer acercamiento al análisis de los datos recabados en las primeras dos entrevistas y por lo tanto de las temáticas planteadas. Este ejercicio resultó en la expansión en la cantidad y calidad de preguntas a utilizar en las siguientes entrevistas, que pertenecen a la segunda fase de la investigación. El listado de preguntas final se agrupó en nueve momentos: a) sobre el involucramiento y motivación del individuo en la realización de actividades de investigación; b) el significado de ser investigador desde su experiencia; c) el sentir y ser del sujeto investigador; d) área de investigación, nivel de dominio y experiencias transdisciplinarias; e) formas de asumir los riesgos y manejar las exigencias de la investigación; f) influencia sobre otros sujetos; g) la formación de investigadores y sus retos; h) experiencias de convivencia y colaboración con otros; e i) definir con una oración y luego con una palabra qué significa ser investigador, para fomentar la reflexión por parte de los entrevistados.

\section{REFERENCIAS}

Boddy, C. (2016). Sample size for qualitative research. Qualitative Market Research, 19(4), 426432. http://doi.or/10.1108/QMR-06-2016-0053.

Cooan, E., Geekie, J., Goldstein, S., Jeskins, L., Jones, R., Macrae-Gibson, R., Secker, J., y Walton, G. (2020). Definición de alfabetización informacional de CuIP, 2018. Anales de Documentación, 23(1), 1-5. http://doi.org/10.6018/analesdoc.373811.

González, F. (2019). La subjetividad y su significación para el estudio de los procesos políticos: sujeto, sociedad y política. En C. Echandía, A. Díaz y P. Vommaro (comps.), Subjetividades politicas: desafíos y debates latinoamericanos (pp. 11-29). Universidad Distrital Francisco José de Caldas. Recuperado de: https://repositorio.idep.edu.co/handle/001/2170.

Hernández, A. (2019). El sistema nacional de investigadores. Tensiones, desafíos y oportunidades para los académicos. Sociológica, 34(98), 85-110. Recuperado de: https://sociologicamexico.azc.uam.mx/index.php/Sociologica/article/view/1571.

Mercado, P., Cernas, D., y Nava, R. (2015). La interdisciplinariedad económico-administrativa en la conformación de una comunidad científica y la formación de investigadores. Revista de Educación Superior, 65(1), 43-65. Recuperado de: http://doi.org/10.1016/j.resu.2015.12.011. 
Olivas, J., y Musi, B. (2019). Evaluación científica y el sistema nacional de investigadores. Cuadernos Fronterizos, 15(46), 44-46. Recuperado de: https://erevistas.uacj.mx/ojs/index. $\mathrm{php} /$ cuadfront/article/viewFile/2827/2803.

Pickard, A. (2013). Research methods in information (2a. ed.). Facet Publishing.

Pinto, M., y Uribe-Tirado, A. (2016). Presencia de formación de competencias informacionales (AlfiN) en las bibliotecas nacionales iberoamericanas. Investigación Bibliotecológica, 31(71), 73-102. http://doi.org/10.22201/iibi.0187358xp.2017.71.57811.

Rodríguez, J., González, C., y Maqueda, G. (2017). El Sistema Nacional de Investigadores en México: 20 años de producción científica en las instituciones de educación superior (19912011). Investigación Bibliotecológica, (esp. bibliometría), 187-219. http://dx.doi.org/10.22201/ iibi.24488321xe.2017.nesp1.57890.

Torres-Frías, J., Moreno-Bayardo, M., y Jiménez-Mora, J. (2018). Aportes de lectores y lectoras de tesis doctoral como mediación pedagógica en la formación de personas investigadoras. Revista Educación, 42(1), 1-21. http:/ /doi.org/10.15517/REVEDU.V42I1.22970.

Vessuri, H. (2007). La formación de investigadores en América Latina. En J. Sebastian (ed.), Claves del desarrollo científico y tecnológico de América Latina (pp. 1-37). Siglo XXI/Fundación Carolina Rodríguez Miramontes.

Cómo citar este artículo:

Ortiz-Santana, T. G., Machin-Mastromatteo, J. D., y Tarango, J. (2020). La formación de nuevos investigadores y el manejo de las alfabetizaciones informacional, científica y académica. Revista Electrónica Científica de Investigación Educativa, 5(1), pp. 45-54. doi: doi.org/10.33010/recie.v5i1.999. 